\title{
Research on Key Technologies of Mode Multiplexing I Demultiplexing
}

\author{
Su Zhiyao ${ }^{1}$ \\ ${ }^{1}$ College of Physics and Optoelectronic Engineering, Shenzhen University \\ Shenzhen, 518061, China
}

\begin{abstract}
The rapid growth of Internet capacity has driven the continuous progress of optical fiber communication technology. The transmission capacity of the current optical fiber communication system is close to the limit of single-mode optical fiber transmission. The mode-division multiplexing technology based on few-mode optical fiber can increase the communication capacity exponentially, which is the current research hotspot. Mode multiplexing and demultiplexing are one of the key technologies to realize modular division multiplexing. This paper expounds the existing mode multiplexing / demultiplexing schemes and their corresponding research progress, and discusses the current problems and future development application prospects of optical fiber communication module division multiplexing technologies.
\end{abstract}

\section{Introduction}

With the accelerated development of information technology and network technology, network services have become increasingly diversified, and new application requirements are constantly emerging. Nowadays, concepts such as "Internet +", "big data", "cloud computing", and "AR" all represent new trends, new technologies, and new industrial forms. Technologies such as "IPTV", "4G" have also gone deep into all aspects of human life. The vigorous development and large-scale application of all these information technologies are accompanied by the generation of large amounts of data. Moreover, with the development of the society, data is continuously and exponentially increasing. It can be said that the current society is already in the age of data. At the same time, what supports these emerging technologies is the optical communications network. The backbone network, metropolitan area network, access network, and Tiandi integrated nJetwork connect each terminal device to share and exchange data with each other. The continuous growth of data means that the demand for optical network capacity is also increasing. In the past few decades, network capacity demand has increased by 10 times every 7 years. Facing the demand of network capacity, it is required to continuously improve the transmission capacity of communication systems or optical fibers [1-3]

The emergence of fiber and semiconductor lasers and optical amplifiers has changed the development direction of the entire communication system. Since then, optical fiber communication technology has continued to develop to meet the growing network capacity requirements, during which several technical reforms have been carried out: time division multiplexing technology, wavelength division multiplexing technology, high-spectrum high-order modulation format, etc. In the 1970s, the advent of optical fibers and semiconductor lasers changed the development direction of the entire communication system. Since then, fiber optic communication technology has continued to develop, thereby meeting the ever-increasing network capacity requirements. By the late 1990s, an optical fiber was able to transmit digital information from hundreds of gigabits per second to terabytes per second. After 2000, the high-order modulation format technology continued to improve the performance of optical communication systems by increasing the spectrum utilization rate to meet network capacity requirements. However, the growth of single-mode optical fiber transmission systems will be limited by Shannon Limit [4], and the capacity limit that can be achieved using existing technology is 100 terabits per second. Since the demand for network capacity is still increasing day by day, in order to expand transmission capacity, it is imperative to study new technologies.

As an effective means to break through the nonlinear Shannon Limit of standard single-mode optical fiber, the origin of space division multiplexing technology can be traced back to the early stage of optical fiber communication. As early as 1979, people produced multi-core optical fibers that can be used in space division multiplexing systems [5]; in 1982, someone used a 10-meter MMF to transmit two fiber modes simultaneously, which verified the feasibility of analog-division multiplexing on short-distance transmission. However, because the capacity of single-mode optical fiber still had great development space at that time, relevant research on space division multiplexing had not received much attention. Until 
recently, with the increasing demand for network capacity, space division multiplexing technology has begun to achieve rapid development. The space division multiplexing technology has many branches, which can be divided into multi-fiber multiplexing, multi-core fiber multiplexing and multi-mode multiplexing, etc. Multi-fiber multiplexing refers to physical parallel spatial channel multiplexing. It consists of multiple parallel single-mode fibers and devices, so it can increase the system capacity exponentially, but the corresponding system overhead and energy loss also increase exponentially. Multi-core multiplexing refers to a single fiber and multiple cores. The signal is propagated in each core of the fiber, which can also double the system capacity, and at the same time minimize the overhead. Modular division multiplexing technology is a kind of space division multiplexing technology, that is, a method of using orthogonality between modes in a multimode fiber to carry different information as independent channels in different modes to propagate simultaneously in the fiber. In an ideal situation, as long as there are enough modes supported by the fiber, the spectrum utilization rate can be greatly improved to meet the greater capacity requirements.

In the mode division multiplexing system, the mode multiplexer / demultiplexer is the most critical part. The mode multiplexer can convert and couple the fundamental mode in a single-mode fiber to multiple different higher-order modes in a few-mode fiber, thereby achieving mode multiplexing. The demultiplexer has a dual function, which can convert different modes into the fundamental mode in single-mode fiber. This paper gives a detailed introduction to the current mainstream mode multiplexer / demultiplexer implementation, and looks forward to the future development trend of the mode multiplexer / demultiplexer.

\section{Mode multiplexer/demultiplexer scheme}

\subsection{Free space optics}

\subsubsection{Phase plate method}

The phase plate is a phase modulation device. By coating the surface of the device, a refractive index difference will occur at different positions, so that the passing beam will have a phase difference so that the input light can be arbitrarily phase-modulated. Figure 1 shows a few-mode multiplexing system based on phase plate and optical beam splitter [6]. After passing through the output port, the Gaussian light output from the single-mode fiber is modulated into a LP11a mode by the phase plate. The modulated LP11a mode and Gaussian light from the 0 output port pass through the optical beam splitter at the same time and are multiplexed together. At the same time, the Gaussian light from the 2 output port is modulated into a PL11a mode after passing through the phase plate and passes through the optical beam splitter and is coupled together with the multiplexed light, thereby achieving the multiplexing of the three modes. When the multiplexing mode needs to be added, only the phase plate corresponding to the mode needs to be increased and the newly generated linear polarization mode and the multiplexed light should pass through the optical beam splitter at the same time. In theory, any mode of modulation and multiplexing can be achieved by using phase plates and optical beam splitters. However, it is worth noting that because the beam splitter has semi-transparent and semi-reflective characteristics, each beam will lose half of the optical power after passing through the beam splitter, anddifferent modes will have different losses according to the number of optical beam splitters passed during multiplexing at the same time.

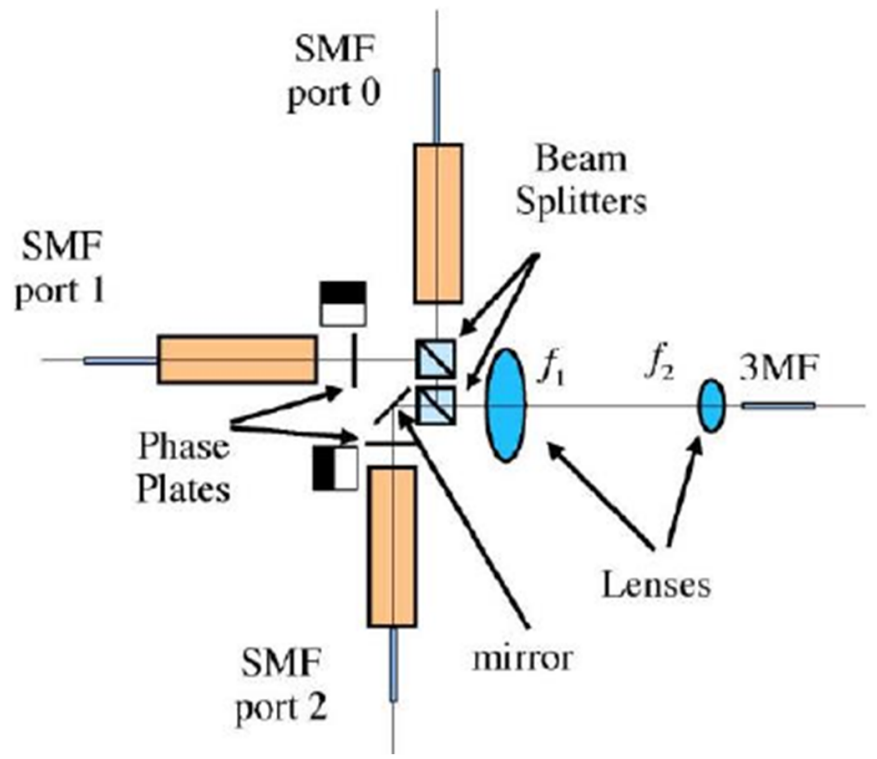

Fig.1 Schematic setup of mode multiplexer

\subsubsection{Spatial light modulating method}

Spatial Light Modulator is a device that modulates the spatial distribution of light waves and is widely used in 
mode multiplexing transmission systems such as low-mode, orbital angular momentum (OAM) and vector mode [7-9]. The spatial light modulator contains many independent units, which are arranged in a one-dimensional or two-dimensional array in space. Each independent unit can be controlled through optical signals or electrical signals, and changes its own optical properties through various physical effects to modulate the input light. Figure 2 is the schematic diagram of the spatial light modulator in practical application[8]. As a diffractive element, LCOS (liquid crystal on silicon) is set on the Fourier transform surface of the 4F optical system to modulate the phase of the lateral light field. The light output from the SMF in the figure is collimated by the left lens, and then passes through a lens. The function of this lens is equivalent to Fourier transform. After spatial phase modulation by LCOS, the light passes through the second lens, which is equivalent to inverse Fourier transform, and its purpose is to optimize the performance of the converter. The focal length of the first lens should match the desired mode. In this way, the required modes can be excited in $\mathrm{MMF}$, and then the modes can be reused. The performance of the LCOS element can be controlled by computer programming to generate different phase modulations of the fundamental mode beam, thereby obtaining different high-order mode fields.

In general, multiple LCOS devices can be used to achieve multiplexing of different modes. However, such a system is generally large in structure and complicated in operation, so it is not easy to put it into practical use. The University of Eindhoven in the Netherlands uses a superimposed blazed grating phase plate as a diffraction device [11]. This method can generate different modes at the same time through one LCOS, and the system composition after LCOS greatly reduces the complexity of the system, and also improves the crosstalk and system losses of the module. The device is shown in Figure 3.

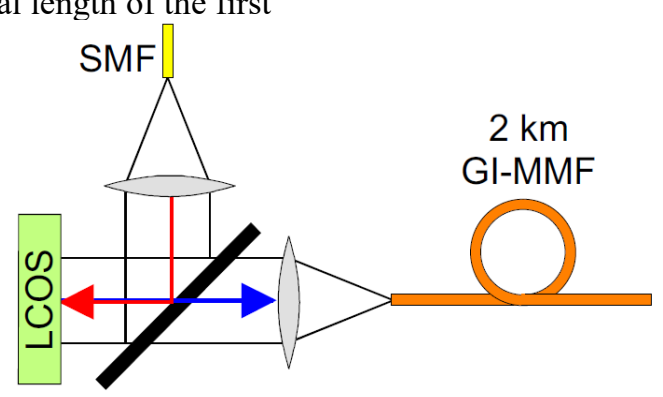

Fig.2 Schematic setup of the spatial light modulator

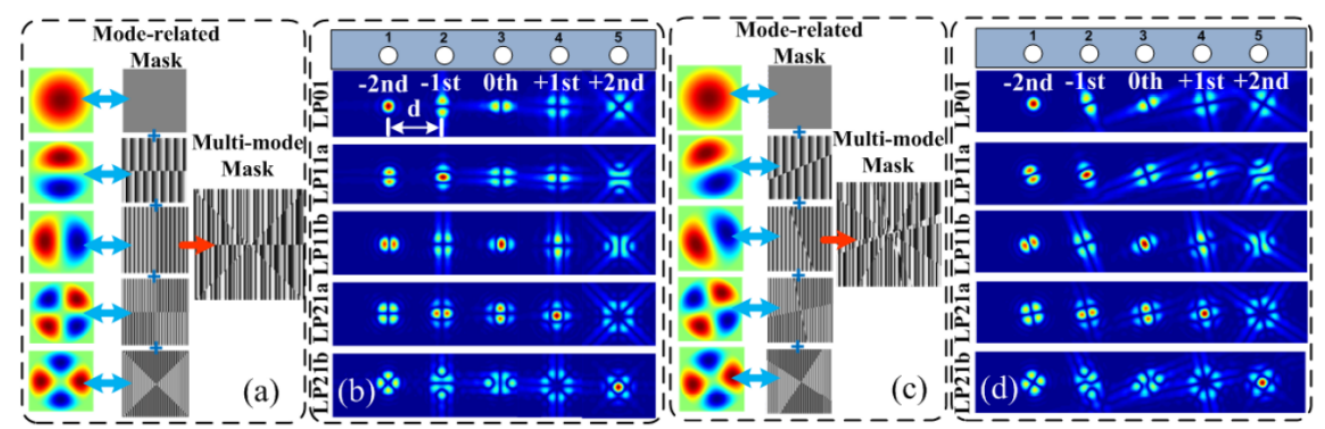

Fig.3 Lcos and mode-related features

\subsection{Guided wave optics method}

\subsubsection{Optical fiber grating+coupler}

Researchers at the University of Melbourne in Australia designed a grating-based mode converter. The working principle of this grating is that the transmission mode in the fiber under stress will be converted, so different long-period fiber gratings carved by pressure are used to complete the mode conversion function. In addition, the research team developed a fusion symmetric coupler to achieve the multiplexing function of the mode. Figure 4 shows the principle diagram based on fiber grating and fusion symmetric waveguide coupler. $\mathrm{MCl}$ realizes the conversion from LP01 mode to LP11 mode, and SMC realizes the multiplexing of LP01 mode and LP11 mode. Based on this mode multiplexing scheme, they successfully completed LP01 and LP11 mode conversion and transmission [12]. The grating structure has the characteristics of low insertion loss and easy integration and coupling, but it is only suitable for low-order mode conversion and cannot be reconstructed. 


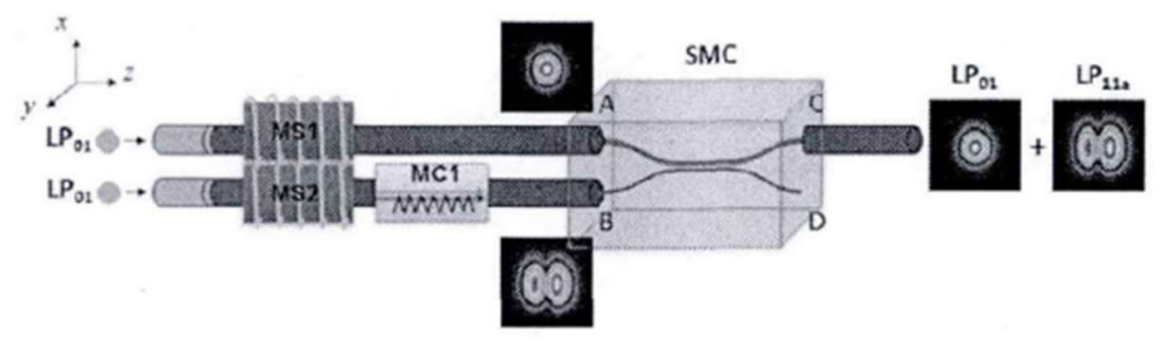

Figure 4: optical fiber grating+coupler principle diagram

\subsubsection{Mode selection coupler}

Fiber type mode selective coupler is an asymmetric direction coupler, which is composed of a pre-tapered single-mode fiber and a few-mode fiber fusion. After passing through the mode selective coupler, the LP01 mode in the single mode fiber will be selectively coupled to a higher order mode in the less mode fiber. The principle of mode coupling is that the LP mode in a single-mode fiber matches a certain higher-order LP mode propagation constant in a few-mode fiber. The mode selection coupler based on the fusion taper is one of the most mature optical fiber device technologies, and has a very good market prospect. The following will introduce a parallel FMF mode multiplexer. With this multiplexer, the complexity of cascading multiple couplers can be avoided [13]. The structure of the device is shown in Figure 5 below. The device consists of three input ports and three output ports, single-mode fiber 1 is connected to input port 1 and output port 4; single-mode fiber 2 is connected to input port 3 and output port 6 ; input port 2 and output Port 5 is connected to a low-mode fiber. The input port inputs the fundamental mode, and each SMF and FMF respectively form a mode coupler. One coupler completes the transition from LP01 to LP11, another coupler completed the conversion from LP01 to LP02. The basic modes input from these three ports can be coupled to the LP01, LP11, and LP02 modes in the FMF based on a reasonable design, and output from port 5 at the same time, and then transmitted to the subsequent optical path. The key to realizing the conversion from the basic mode of SMF to the higher-order mode of FMF is to achieve the phase matching condition, that is, to regain the effective refractive index of the basic mode LP01 of SMF1 and SMF2.

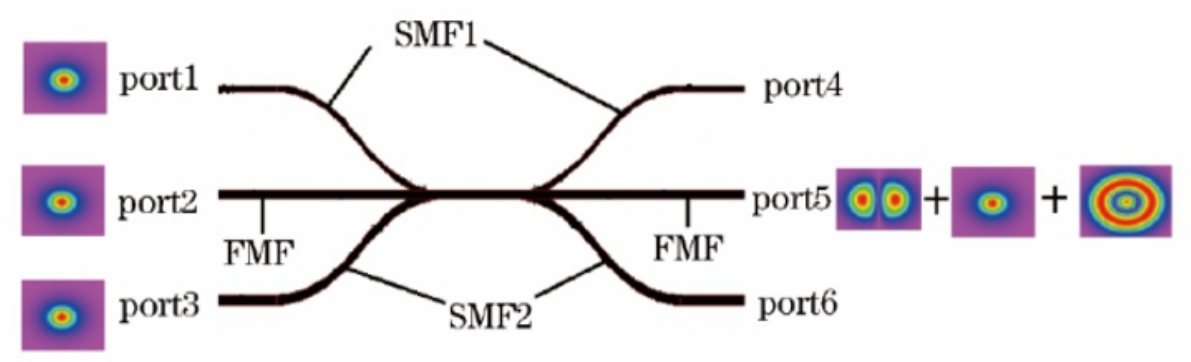

Fig.5 Schematic of mode multiplexer based on cascaded MSCs.

\subsubsection{Photon lantern}

Photon Lantern provides a single-component multiplexer/demultiplexer for mode division multiplexing using FMF. Unlike alternative methods, photonic lanterns are manufactured in the same medium as fiber transmission and provide the best performance in a single component in a compact format. Phoenix Photonics uses in-house proprietary technology to manufacture photonic lanterns to provide the best performance. Provide 3 or 6 fiber inputs, Photonic Lantern is compatible with FMF on the market. According to the researches in recent years, the photon lantern multiplexer / demultiplexer has been widely studied because of its simplicity in structure and high efficiency in achieving mode conversion and mode coupling, and can greatly improve the integration of devices. Compared with discrete-component mode division multiplexers, photon lantern multiplexers can effectively reduce the structural complexity, achieve mode conversion and high-efficiency mode multiplexing, and improve device integration. The structure of the fiber fusion cone photon lamp is shown in Figure 6 [14]. First, special treatment is performed on the three single-mode optical fibers to promote different cladding and core diameters. Then, embed the three optical fibers in the same ferrule. The photon lantern structure can be obtained by melting the taper of the sleeve under adiabatic standards. The core of the processed single-mode fiber is very thin, so the original guided mode light field will leak into the cladding. Since the refractive index of the quartz sleeve is smaller than the refractive index of the single-mode fiber cladding, the distribution of the refractive index enables the fiber cladding and the fused taper quartz sleeve to form a new waveguide structure, thereby ensuring that the optical signal is encapsulated in the fiber In the stable transport 
layer. After a special design, the photon lantern can convert the light field of different single-mode optical fibers into a specific high-order polarization mode and enter the lower-mode optical fiber for transmission, thereby achieving multiplexing of multiple modes. Due to the difficulty of the melt taper process, it is difficult to control the refractive index distribution within the coupling range. [15]. This not only solves the shortcomings of difficult refractive index distribution control, but also improves the repeatability and stability of photonic lanterns, so this method has good prospects for scientific research.
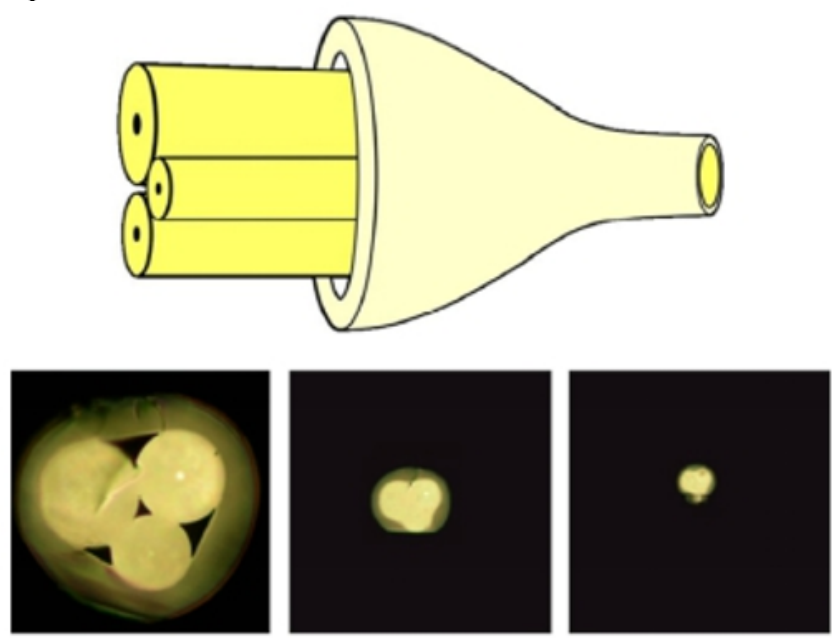

Fig.6 Schematic mode multiplexer made by fusing and tapering three SMFs in and F-doped capillary.

\subsubsection{Mode multiplexer based on silicon-based integration}

The silicon-based integrated mode multiplexing device is one of the key devices for ultra-high-speed on-chip optical interconnects. Traditional waveguide mode multiplexing device is subject to mode coupling mechanisms such as "insulation evolution" or "phase matching". The device is characterized by big size and approaching "minimum length limit", which is the key bottleneck of for the mode multiplexer based on ruled waveguide in the optical interconnection application. For multiplexing schemes based on multimode interference (MMI), asymmetric directional coupler (ADC), and asymmetric Y-branch traditional waveguide modes, as the number of modes increases, the size often reaches hundreds to thousands of microns, thus being unable to meet the needs of ultra-high integration photonic chips.

The difference between ADC and ordinary directional coupler lies in its asymmetric. The plan proposed in 2015 showed that the design of waveguides with different widths is its main principle, which can help to obtain the effective refractive index matching relationship between different modes, thereby achieving mode conversion and multiplexing. [16]. Later, Yunhong Ding and his team proposed a new type of multiplexer in 2013, which completed TE0 and TE1 mode multiplexing [17]. The minimum insertion loss of the device is $0.3 \mathrm{~dB}$, and the crosstalk is less than $-16 \mathrm{~dB}$ in the $100 \mathrm{~nm}$ bandwidth. In addition, through using the ADC mode converter/multiplexer, Dai completed four modes, which included TM0, TM1, TM2, and TM3 multiplexing. Therefore, the ADC can support more multiplexing modes. However, the processing tolerance of the TE mode ADC is very small, which limits its application prospects. While the mode converter/multiplexer based on the MRR structure improves the above-mentioned ADC structure to a certain extent. The MRR structure introduces a micro-ring structure in the asymmetric waveguide, which is equivalent to adding a resonance mechanism. This change increases the flexibility of the device, because the MRR requires less coupling length than the ADC, which improves the complexity of the process, it is more suitable for WDM systems. Chang Weijie proposed an asymmetric Y-branch new mode multiplexer based on sub-wavelength structure [18]. The scheme utilizes the flexible refractive index tailoring of the sub-wavelength structure and the ability to adjust the phase of the light field at the sub-wavelength size, and uses the reverse design method to break through the constraints of the conventional basic mechanism of the adiabatic evolution of the Y-branch waveguide, thereby achieving s new structure of sub-wavelength Y-branch with low radiation loss and large branch angle. The size of the mode multiplexer is reduced by two orders of magnitude. In addition to this, the multiplexing of these two and three modes is realized in sizes of $2.4 \times 3 \mu \mathrm{m} 2$ and $3.6 \times 4.8 \mu \mathrm{m} \mathrm{2}$, respectively. This is also the smallest size reported internationally. This shows that they have broad application prospects in ultra-large-scale integrated silicon-based optical interconnects.

\section{Summary}

This article introduces two major categories of mode division multiplexed devices-mode division multiplexer based on waveguide optics and mode division multiplexer based on spatial optics. The advantage of the device based on space optics is free space and flexible design, but the requirements for manual collimation are relatively high. In addition, the space optics method uses more optical components, resulting in greater insertion loss and coupling loss. Future research should focus on 
developing new methods to reduce these losses. The features of the waveguide optical method are its high integration, the large number of modes it can carry, and its transmission capacity is greatly improved compared to the spatial optical method. Although the insertion loss and coupling loss of this device are very small and the delay is not large, the technology and algorithm requirements for demultiplexing are very high, and the requirements for the manufacturing process are also very strict. Due to the development of silicon-based technology in recent years and the requirement for highly integrated devices, the silicon-based mode division multiplexer is undoubtedly the most prospective.

\section{Acknowledgements}

First and foremost, I am most grateful to my supervisor, whose useful suggestions, incisive comments and constructive criticism have contributed greatly to the completion of this thesis. She devotes a considerable portion of her time to reading my manuscripts and making suggestions for further revisions. Her in having gone through the draft versions of this thesis several times as well as her great care in life deserve more thanks than I can find words to express.

I am also greatly indebted to all my teachers who have helped me directly and indirectly in my studies. Any progress that I have made is the result of their profound concern and selfless devotion.

\section{References}

1. Agrawal D., Tarokh V., Naguib A., et al. Space-time coded OFDM for high data-rate wireless communication over wideband channels[C] Space-time coded OFDM for high data-rate wireless communication over wideband channels.

2. Huang Ming Fang, Qian Dayou, Ip E. 50.53-Gb/s PDM-1024QAM-OFDM transmission using pilot-based phase noise mitigation[C] $50.53-\mathrm{Gb} / \mathrm{s}$ PDM-1024QAM-OFDM transmission using pilot-based phase noise mitigation. ,

3. Darwazeh I, Xu Tongyang, Gui Tao, et al. Optical SEFDM System; Bandwidth Saving Using Non-Orthogonal Sub-Carriers[J]. IEEE Photonics Technology Letters, 2014, 26(4): 352-355.

4. Essiambre Renéjean, Kramer Gerhard, Winzer Peter J., et al. Capacity Limits of Optical Fiber Networks[J]. Journal of Lightwave Technology, 2010, 28(4): 662-701.

5. Iano S., Sato T., Sentsui S., et al. Multicore optical fiber[J]. Lightwave, 1979, 321-352.

6. Ryf, Roland, et al. "Mode-Division Multiplexing Over $96 \mathrm{~km}$ of Few-Mode Fiber Using Coherent $6 \$$, ,times $\backslash, \$ 6$ MIMO Processing." Journal of Lightwave technology 30.4 (2011): 521-531.

7. Gibson G, Courtial J, Padgett M, et al. Free-space information transfer using light beams carrying orbital angular momentum[J]. Optics Express, 2004, 12(22): 5448.

8. Willner A. E., Huang H., Yan Y., et al. Optical communications using orbital angular momentum beams[J]. Advances in Optics \& Photonics, 2015, 7(1): 66-106.

9. Feng Yuan Hua, Luo Jiawei, Li Yuru, et al. Mode Multiplexing and High Efficient Switching in Few-Mode Fiber Based on Modeled Blazed Grating[J]. IEEE Photonics Journal, 2016, 8(3): 1-7.

10. Chen, Haoshuo, and Ton Koonen. "Single multi-mode mask for multi-channel mode division demultiplexing." Optical Fiber Communication Conference. Optical Society of America, 2013.

11. B Franz, H Bülow. Mode group multiplexing over graded-In- dex multimode fiber. [C] IEEE ICTON( 2012), Th.A1.3.

12. Li, An, et al. "Fused fiber mode couplers for few-mode transmission." IEEE Photonics Technology Letters 24.21 (2012): 1953-1956.

13. Le Yansi, Wang Zhi, Li Qiang et al, Research on Optical Fiber Three-Mode Multiplexer and Demultiplexer[J], China Lase, 2016(6):185-190.

14. Chen H, Fontaine N K, Ryf R, et al. Design constraints of photonic-lantern spatial multiplexerbased on laser-inscribed 3-D waveguide technology[J]. Journal of Lightwave Technology, 2015,33(6):1147-1154.

15. Greenberg, Maxim, and Meir Orenstein. "Multimode add-drop multiplexing by adiabatic linearly tapered coupling." Optics express 13.23 (2005): 9381-9387.

16. Ding, Yunhong, et al. "On-chip two-mode division multiplexing using tapered directional coupler-based mode multiplexer and demultiplexer." Optics express 21.8 (2013): 10376-10382.

17. Dai, Daoxin, Jian Wang, and Yaocheng Shi. "Silicon mode (de) multiplexer enabling high capacity photonic networks-on-chip with a single-wavelength-carrier light." Optics letters 38.9 (2013): 1422-1424.

18. W. Chang, et al. "Ultra-compact mode (de) multiplexer based on subwavelength asymmetric Y-junction,” Opt Express. 26(7),8162-8170(2018). 